\title{
The time course of the verbal prediction effect
}

\author{
ENRIQUE ACOSTA, JR. \\ University of Wyoming, Laramie, Wyoming 82071 \\ and \\ JAMES V. HINRICHS \\ University of Iowa, Iowa City, Iowa 52240
}

\begin{abstract}
Choice reaction times following correct verbal predictions of the presented stimulus are faster than after incorrect predictions. Two experiments examined the influence of the time interval (PSI) between the prediction and the stimulus on the magnitude of the prediction effect. Experiment 1 used equiprobable stimuli and showed a smaller prediction effect at a 2-sec PSI than at a 0- or .5-sec PSI. Experiment 2 used unequal-probability stimuli and showed that the prediction effect was independent of PSI when the more frequent stimulus was predicted. When the less frequent stimulus was predicted, however, the magnitude of the prediction effect was smaller at .75- and 2-sec PSIs than at a 0 -sec PSI. These results were discussed in terms of a prediction substitution hypothesis.
\end{abstract}

In recent years, studies concerned with selective preparation in information processing have made extensive use of the verbal prediction paradigm. A verbal prediction task superimposes the subject's hypothesis of a to-be-presented stimulus on a choice reaction time (RT) task. These hypotheses, or verbal predictions, are assumed to correspond to the subject's covert expectation for one of several stimuli (Bernstein \& Reese, 1965; Hinrichs, 1970). The usual finding when such a procedure is employed is that RTs to correctly predicted stimuli are faster than RTs to incorrectly predicted stimuli (e.g., Bernstein \& Reese, 1965). The early research using verbal predictions concentrated on the concept of expectancy (and its inference through verbal predictions) as an explanatory construct of empirical phenomena such as stimulus probability effects (Geller, Whitman, \& Post, 1973; Hinrichs, 1970) and repetition effects (Geller \& Pitz, 1970; Keele, 1969) on choice RT tasks.

In the case of stimulus probability, Hinrichs (1970) has argued that a possible interpretation of the probability effect (faster RTs to the more probable stimulus) is that RTs to the more probable stimulus are a mixture of expected (correctly predicted) and unexpected (incorrectly predicted) events. His results showed that, using a $2: 1$ stimulus frequency ratio, the overall probability effect disappeared when the data were conditionalized by subject's prediction outcome (correct or incorrect). These results are equivocal. For example, Hinrichs and Craft (1971) required their subjects to predict the expected stimulus under a

Requests for reprints should be sent to Enrique Acosta, Jr., who is now at the Psychology Department, University of Wyoming, P.O. Box 3415, University Station, Laramie, Wyoming 82071 . number of stimulus frequency ratios $(1: 1$ to $9: 1)$ and found residual probability effects after conditionalizing the data by prediction outcome. Similar findings have been obtained by Geller et al. (1973) and by Whitman and Geller (1972). In the case of stimulus repetition, Keele (1969) found an absence of repetition effects (faster RTs to repetitions of a stimulus than to alternations) when the data were conditionalized by prediction outcome. Geller and Pitz (1970) obtained similar results.

One area of investigation that has been largely neglected by researchers in verbal predictions concerns the effects of the time interval between a prediction and a stimulus presentation (PSI) on the magnitude of the prediction effect. The usual procedure allows this time interval to vary randomly either by requiring the experimenter to manually initiate the stimulus presentation after the subject's prediction (e.g., Geller, 1974), or, in the case of automated stimulus presentations, by using an intertrial interval sufficiently long (e.g., $4 \mathrm{sec}$ ) to allow the subject to make a prediction (e.g., Hacker \& Hinrichs, 1974). The assumption implicit in either procedure is that, once a verbal prediction has been uttered, it will remain in memory, unchanged, until the stimulus in presented.

Geller and Whitman (1972) have challenged this assumption by varying the time between a subject's prediction and the stimulus from 3 to $11 \mathrm{sec}$. Their results showed that, as the PSI increased, the difference in RT between correctly and incorrectly predicted stimuli decreased, that is, a Prediction Outcome by PSI interaction. Geller and Whitman attributed these results to a fading of the memory trace of the prediction as the PSI increased. Holender and Bertelson (1975) conducted two experiments in which the PSI varied between 1.5 and $6 \mathrm{sec}$ in one (Experiment 2) and between 4.0 and $12.5 \mathrm{sec}$ in the other (Experiment 3 ). These investigators 
found a reduction in the effect of prediction outcome as the PSI increased in their second experiment, although the interaction failed to reach an acceptable level of significance $(.05<\mathrm{p}<.07)$. In Experiment 3 , however, Holender and Bertelson failed to find a reduction in the effect of prediction outcome as the PSI increased.

It should be noted, however, that Holender and Bertelson's (1975) procedure included the presentation of a warning signal and a variable foreperiod between the prediction and the stimulus. Thus, it is impossible to determine if the lack of a Prediction Outcome by PSI interaction found in Holender and Bertelson's experiments was due to: (1) additive effects of prediction outcome and foreperiod, or (2) the presence of the warning signal. In addition, the PSIs in Holender and Bertelson's experiments are only approximations since, in their second experiment, "the warning signal was set on approximately $1 \mathrm{sec}$ after S's prediction" (p. 197) and, in their third experiment, a prediction light instructed the subject to make a prediction. In the latter case, the calculated PSIs $(4.0$ to $12.5 \mathrm{sec}$ ) are the sum of the prediction light/warning signal interval and the warning signal/stimulus onset interval. Thus, in their second experiment, the experimenter's RT was a component of the PSI, while, in their third experiment, the subject's RT was a component of the PSI. A simpler procedure with which to study the joint influence of prediction outcome and PSI on decision making would be to exclude the presentation of any signals during the PSI, as Geller and Whitman (1972) did. The inclusion of a warning signal in this time interval may be unnecessary since, as Posner and Boies (1971) have noted, primes (or, in this case, predictions) have a general alerting function in addition to their specific effects.

Although Geller and Whitman's (1972) and Holender and Bertelson's (1975) studies differ in a number of ways, they both examined PSIs that are longer than those used in most of the prediction research (between 2 and $4 \mathrm{sec}$ ). Thus, the goal of the present studies is to examine PSIs that are shorter than those used in most prediction studies. If, within this range, verbal predictions were maintained unchanged in memory, then the magnitude of the prediction effect should be independent of PSI. If, on the other hand, verbal predictions in memory were subject to changes, then the magnitude of the prediction effect should decline as the PSI is increased.

\section{EXPERIMENT 1}

The first experiment was designed to determine the effects of systematic variations in PSI on the prediction effect. The present research differs from that of Geller and Whitman (1973) and Holender and Bertelson (1975) in that the PSIs used $(0, .5,1.0$, and $2.0 \mathrm{sec})$ are shorter than those used in typical prediction research (about $2-4 \mathrm{sec})$.

\section{Method}

Subjects. The subjects were four right-handed females recruited from an introductory psychology course at the University of Iowa.

Apparatus. The apparatus measured choice RT to a series of light stimuli. The subject's task was to press a left- or right-hand key in response to a red or green light. The subject's verbal prediction activated a voice-operated relay (BRS Foringer VOR001), which, in turn, activated a waiting circuit corresponding to the PSI programmed for that trial. The experimenter recorded the subject's verbal prediction on every trial. The stimulus sequence (light and PSI) was punched on paper tape that was read by a perforated tape reader. RTs (in milliseconds) and the responses were recorded on paper tape. Digital logic provided overall control of stimulus presentation and data acquisition.

Procedure and Experimental Design. Each subject was administered four blocks of trials. Each block consisted of 16 warm-up trials and 136 test trials. The red and green lights, as well as the four different values of PSI, were equally represented within each block of trials. A trial consisted of the presentation of a "predict" light followed by the subject's verbal prediction. The subject's prediction triggered a voice-operated relay connected to a throat microphone that turned off the predict light and started the PSI of $0, .5,1.0$, or $2.0 \mathrm{sec}$. After the preprogrammed PSI had elapsed, the red or green stimulus light was presented. The subject's response turned off the stimulus light and, $1.5 \mathrm{sec}$ later, the predict light for the following trial was presented.

\section{Results and Discussion}

Median RTs were calculated for each of the eight treatment combinations of prediction outcome and PSI for each block for each subject. Trials in which response errors were made (1.2\% of all trials) were excluded from these calculations.

Table 1 shows the means of median RTs as a function of PSI and prediction outcome. An analysis of variance of these data included outcome, PSI, and blocks as factors. The significant effect of outcome $[F(1,3)=50.44$, $\mathrm{p}<.01]$ replicates the typical finding that $\mathrm{RT}$ to correctly predicted stimuli is faster than RT to incorrectly predicted stimuli ( 436 vs. $583 \mathrm{msec}$ ). The main effect of PSI $[F(3,9)=22.55, p<.01]$ suggests that a verbal prediction has a general effect analogous to a warning signal. At the 0-sec PSI, RT was approximately $100 \mathrm{msec}$ slower than at the .5-, 1.0-, and 2.0-sec PSIs ( 585 vs. 489,474 , and $490 \mathrm{msec}$ ).

The interaction between prediction outcome and PSI was also significant $[F(3,9)=5.41, p<.05]$. Follow-up $1 \mathrm{deg}$ of freedom contrasts indicated that the magnitude of the prediction was greater at the 0- and .5-sec PSIs (156 msec and $172 \mathrm{msec})$ than at the 2-sec PSI

Table 1

Means and Standard Errors (SE) of Median Reaction Times as a Function of Prediction Outcome and PSI

\begin{tabular}{|c|c|c|c|c|c|c|c|c|c|}
\hline \multirow{3}{*}{$\begin{array}{c}\text { Prediction } \\
\text { Outcome }\end{array}$} & \multicolumn{9}{|c|}{ PSI (in Seconds) } \\
\hline & \multicolumn{2}{|c|}{0} & \multicolumn{2}{|c|}{.5} & \multicolumn{2}{|c|}{1.0} & \multicolumn{2}{|c|}{2.0} & \multirow[b]{2}{*}{ Mean } \\
\hline & Mean & SE & Mean & SE & Mean & $\mathrm{SE}$ & Mean & SE & \\
\hline $\begin{array}{l}\text { Correct } \\
\text { Incorrect }\end{array}$ & $\begin{array}{l}507 \\
663\end{array}$ & $\begin{array}{l}24 \\
35\end{array}$ & $\begin{array}{l}403 \\
575\end{array}$ & $\begin{array}{l}14 \\
19\end{array}$ & $\begin{array}{l}401 \\
548\end{array}$ & $\begin{array}{l}12 \\
21\end{array}$ & $\begin{array}{l}434 \\
547\end{array}$ & $\begin{array}{l}17 \\
17\end{array}$ & $\begin{array}{l}436 \\
583\end{array}$ \\
\hline Mean & 585 & & 489 & & 474 & & 490 & & \\
\hline
\end{tabular}


$(113 \mathrm{msec}) \quad[F(1,9)=8.13$ and 15.30 , respectively, both $\mathrm{ps}<.05]$. This interaction is indicative of a functional relation between PSI and the magnitude of the prediction effect. No other main effects or interactions were significant. The smaller prediction effects found at the longest PSI suggest that the memory representation of the verbal prediction is subject to change. One interpretation of this spontaneous change is that the memory representation of the predicted event decays over time (Geller \& Whitman, 1972).

An alternative interpretation of the results of Experiment 1 is consistent with an attention-switching notion (cf. Shiffrin \& Schneider, 1974, p. 626). Specifically, on some proportion of trials, a subject substitutes an expectation for a particular stimulus (e.g., red) with an expectation for the other stimulus (in this case, green). The result of this substitution is that RT to (nominally) correct predictions (i.e., a red light is presented) will increase and RT to (nominally) incorrect predictions (i.e., a green light is presented) will decrease. Thus, this mixture of correct and incorrect predictions results in a reduction in the magnitude of the prediction effect. Since it is expected that the substitution operation would entail a finite amount of time, it is not unreason. able to expect the decrease in the magnitude of the prediction effect to occur only at PSIs sufficiently long to allow the substitution operation to occur.

\section{EXPERIMENT 2}

One immediate application of the results of Experiment 1 and the substitution hypothesis concerns the expectancy interpretation of the stimulus probability effect. Falmagne (1965) and, more recently, Lupker and Theios $(1975,1977)$ have argued that the underlying process in choice RT tasks involving unequiprobable stimuli is a two-state process depending on whether the subject is prepared for the stimulus presented on a given trial or unprepared for that stimulus. If a subject is prepared for the presented stimulus, then RT to that stimulus will be faster than if the subject is unprepared. Hinrichs (1970) viewed the verbal prediction paradigm as a possible indicator of these subjective expectancies or preparations. The expectancy interpretation predicts that probability effects would disappear when the data were conditionalized by the subject's prediction outcome. In fact, a considerable proportion of the variance attributable to the probability effect is removed by conditionalization (Hinrichs \& Craft, 1971). However, a number of studies (Geller, 1974; Geller et al., 1973; Hinrichs \& Craft, 1971; Whitman \& Geller, 1973) found residual probability effects after conditionalizing the data on prediction outcome. These residual probability effects can be viewed as either: (1) faster RTs to the more frequent stimulus independent of prediction outcome, or (2) a smaller prediction effect for the less frequently predicted stimulus than for the more frequently predicted stimulus. A possible interpre- tation of the residual probability effect is that these residual probability effects may be artifacts produced by differential rates of substitution for the more frequently predicted and the less frequently predicted stimuli. Specifically, when a subject predicts the more frequent stimulus, there is a large probability that the prediction will be confirmed. Thus, there is little reason for a subject to switch predictions. However, when the less frequent stimulus is predicted, there is a large probability that the prediction will not be confirmed and, consequently, covert substitutions will result in a "fast" response. By this line of reasoning, the substitution hypothesis predicted that the magnitude of the prediction effect should decrease with PSI at a faster rate when the less frequent stimulus is predicted than when the more frequent stimulus is predicted. In addition, at PSIs too short to allow any substitutions $(e . g ., 0 \mathrm{sec})$, the magnitude of the prediction effect should be the same for both the more and the less frequently predicted stimuli (i.e., residual probability effects should be absent at the 0-sec PSI). Experiment 2 tested these predictions.

In Experiment 2, the relative frequencies of the stimulus events were .75 and .25 . In order to obtain an adequate number of observations for the less frequently predicted stimuli, the number of PSIs was reduced to three $(0, .75$, and $2 \mathrm{sec})$. In addition, a control block that did not require verbal predictions was included in the design in order to examine the joint influence of PSI and stimulus probability uncontaminated by verbal predictions.

\section{Method}

Subjects. The subjects were eight females from an introductory psychology course at the University of Iowa. All subjects were right-handed.

Apparatus. The apparatus was essentially the same as that used in Experiment 1. The only major difference was that, instead of red and green lights, the digits 1 and 2 were used as stimuli. The digits were presented with a Monsanto MDA 111 alphanumeric display.

Procedure and Experimental Design. Each subject was administered four blocks of trials, three prediction blocks and one no-prediction block. Each prediction block consisted of 12 warm-up trials and 240 data trials. Prior to the first block of trials, a short ( 12 trials) practice sequence was administered to familiarize the subjects with the procedure. A trial consisted of a click that informed the subjects that a prediction would be accepted followed by the subject's verbal prediction. The subject's prediction triggered a voice-operated relay connected to a throat microphone that started the PSI of $0, .75$, or $2 \mathrm{sec}$. After the preprogrammed PSI had elapsed, the stimulus digit was presented. The subject's response turned off the stimulus digit and, $1.5 \mathrm{sec}$ later, the click for the following trial was presented. One of the digits was presented $75 \%$ of the time, and the other was presented $25 \%$ of the time. The three PSIs were distributed equally among the stimulus alternatives. Thus, for each block of trials, each PSI was followed by the more frequent digit on 60 trials, and each PSI was followed by the less frequent digit on 20 trials. The hand used to respond to the more or less frequent digit was counterbalanced across subjects but ignored in the analysis.

In the no-prediction blocks, the subjects were instructed to say the word "go" after they heard the click. The order of the 
prediction and no-prediction blocks was counterbalanced across subjects.

\section{Results and Discussion}

Median RTs were computed for each subject for each of the experimental conditions of interest: prediction outcome, stimulus predicted, and PSI. Trials in which response errors occurred (.5\% of all trials) were excluded from these computations. The means of median RTs, as well as the standard errors of these means, are presented in the upper half of Table 2.

An analysis of variance on these data included as within-subjects factors prediction outcome, stimulus predicted, and PSI. The order of the prediction and noprediction blocks was included as a between-subjects factor. The analysis revealed a main effect of prediction outcome $[F(1,4)=18.13, p<.02]$, indicating that correctly predicted stimuli were responded to faster than incorrectly predicted stimuli (485 vs. $589 \mathrm{msec}$ ). The main effect of PSI was also significant $[F(2,8)=10.19$, $\mathrm{p}<.01]$. As can be seen in the upper half of Table 2, mean RTs at the 0-sec PSI (638 msec) were slower than the mean RTs at either the .75 -sec PSI $(482 \mathrm{msec})$ or the 2 -sec PSI $(492 \mathrm{msec})$. In addition, the Prediction Outcome by Predicted Stimulus interaction was significant $[F(1,4)=21.26, p<.01]$. This interaction indicated that the magnitude of the prediction effect was larger when the more frequent stimulus was predicted $(156 \mathrm{msec})$ than when the less frequent stimulus was predicted $(52 \mathrm{msec})$.

The Prediction Outcome by Predicted Stimulus by PSI interaction was also significant $[F(2,8)=8.27$, $p<.02]$. Follow-up $1 \mathrm{deg}$ of freedom contrasts $(.05$ level of significance) revealed that the magnitude of the prediction effect when the more frequent stimulus was predicted was independent of PSI $(150,162$, and $155 \mathrm{msec}$ for the $0-, .75$-, and 2-sec PSIs, respectively). The magnitude of the prediction effect when the less frequent stimulus was predicted declined from the 0 -sec PSI $(78 \mathrm{msec})$ to the .75 -sec PSI $(38 \mathrm{msec})$ and remained constant as the PSI increased to $2 \mathrm{sec}$ $(41 \mathrm{msec})$. At the 0 -sec PSI, the magnitude of the prediction effect was significantly larger when the more frequent stimulus was predicted than when the less frequent stimulus was predicted (150 vs. $78 \mathrm{msec})$. In other words, residual probability effects were obtained at the 0-sec PSI.

An analysis of variance was performed on the data obtained from the no-prediction blocks. The analysis included stimulus probability and PSI as within-subjects factors and order of the prediction and no-prediction blocks as a between-subjects factor. The analysis revealed significant main effects of probability $[\mathrm{F}(1,4)=12.04]$ and PSI $[F(2,8)=5.27$, both ps $<.05]$. The main effect of probability indicated that RTs to the more probable stimulus were faster than RTs to the less probable stimulus (558 vs. $497 \mathrm{msec}$ ). The main effect of PSI indicated that RTs at the 0-sec PSI $(609 \mathrm{msec})$ were significantly longer than RTs at either the .75-sec PSI $(487 \mathrm{msec})$ or the 2 -sec PSI $(486 \mathrm{msec})$. No other main effects or interactions were significant (all ps >.10).

\section{GENERAL DISCUSSION}

Two experiments were conducted to determine the effects of temporal variations between a prediction and a stimulus on the magnitude of the prediction effect. What little literature is available addressing this issue has produced equivocal results. Holender and Bertelson (1975) showed prediction effects to be independent of PSI, whereas Geller and Whitman (1972) showed a decrease in the magnitude of the prediction effect with increasing PSI. The present research differs from that of Geller and Whitman in that the decrease in the prediction effect with increasing PSI occurred with

Table 2

Means and Standard Errors (SE) of Median Reaction Times as a Function of Prediction Outcome, Stimulus Predicted, and PSI (Prediction Blocks), and for Stimulus Presented and PSI (No-Prediction Blocks)

\begin{tabular}{|c|c|c|c|c|c|c|c|}
\hline \multirow[b]{3}{*}{ Conditions } & \multicolumn{6}{|c|}{ PSI (in Seconds) } & \multirow[b]{3}{*}{ Mean } \\
\hline & \multicolumn{2}{|c|}{0} & \multicolumn{2}{|c|}{.75} & \multicolumn{2}{|c|}{2.0} & \\
\hline & Mean & SE & Mean & $\mathrm{SE}$ & Mean & SE & \\
\hline & \multicolumn{7}{|c|}{ Prediction Blocks } \\
\hline $\begin{array}{l}\text { More Frequent Predictions } \\
\text { Correct } \\
\text { Incorrect }\end{array}$ & $\begin{array}{l}565 \\
715\end{array}$ & $\begin{array}{l}63 \\
64\end{array}$ & $\begin{array}{l}408 \\
570\end{array}$ & $\begin{array}{l}15 \\
28\end{array}$ & $\begin{array}{l}420 \\
575\end{array}$ & $\begin{array}{l}15 \\
26\end{array}$ & $\begin{array}{l}464 \\
620\end{array}$ \\
\hline $\begin{array}{l}\text { Less Frequent Predictions } \\
\text { Correct } \\
\text { Incorrect }\end{array}$ & $\begin{array}{l}597 \\
675\end{array}$ & $\begin{array}{l}62 \\
51\end{array}$ & $\begin{array}{l}456 \\
493\end{array}$ & $\begin{array}{l}20 \\
17\end{array}$ & $\begin{array}{l}465 \\
506\end{array}$ & $\begin{array}{l}16 \\
20\end{array}$ & $\begin{array}{l}506 \\
558\end{array}$ \\
\hline \multirow[t]{2}{*}{ Mean } & 638 & & 482 & & 492 & & \\
\hline & \multicolumn{7}{|c|}{ No-Prediction Blocks } \\
\hline $\begin{array}{l}\text { More Frequent Stimulus } \\
\text { Less Frequent Stimulus }\end{array}$ & $\begin{array}{l}584 \\
634\end{array}$ & $\begin{array}{l}74 \\
59\end{array}$ & $\begin{array}{l}450 \\
524\end{array}$ & $\begin{array}{l}16 \\
30\end{array}$ & $\begin{array}{l}456 \\
517\end{array}$ & $\begin{array}{l}17 \\
30\end{array}$ & $\begin{array}{l}497 \\
558\end{array}$ \\
\hline Mean & 609 & & 487 & & 486 & & \\
\hline
\end{tabular}




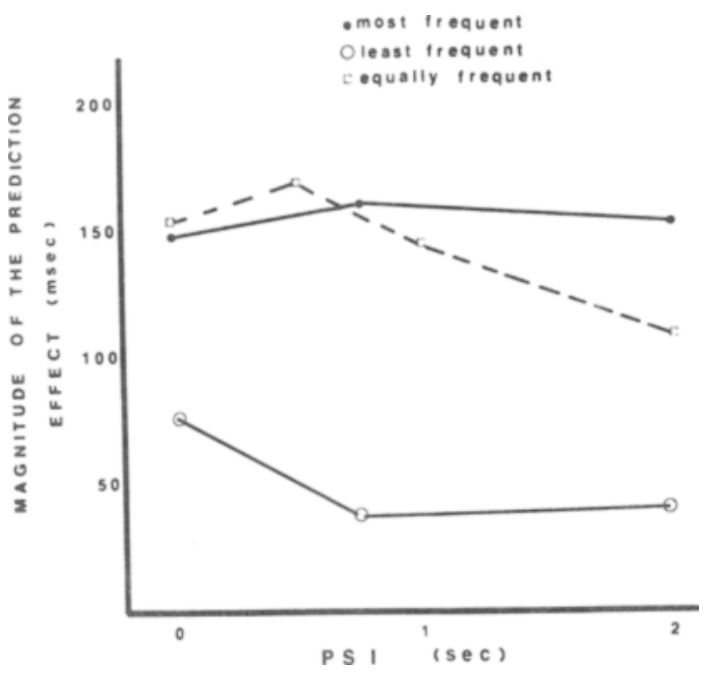

Figure 1. The time course of the prediction effect as a function of prediction frequency for Experiment 1 (dashed line) and Experiment 2 (solid line).

PSIs of $2 \mathrm{sec}$, whereas Geller and Whitman detected a decrease in the prediction effect at a much longer PSI $(7 \mathrm{sec})$. The magnitude of the prediction effect as a function of PSI for Experiments 1 and 2 are shown in Figure 1. Thus, on empirical grounds alone, variations in the time interval between a verbal prediction and a stimulus seem to be an important consideration.

In a more theoretical vein, the results of the first experiment suggested that the internal representation of a verbal prediction does not remain static and unchanged. Rather, the internal representation of a prediction either decays or is interfered with on a certain proportion of trials. The interfering event could be the covert prediction of the unpredicted stimulus. In the first experiment, for example, if a subject predicted red and, prior to the presentation of the stimulus, switched the prediction to green, then RT to a green stimulus on that trial would be faster than RT to a red stimulus. The net result of this strategy would be a reduction in the magnitude of the prediction effect.

The substitution hypothesis was examined in an unequal stimulus probability task in Experiment 2. If a subject does not switch predictions of the more frequent stimulus but switches predictions of the less frequent stimulus, then three effects are expected: (1) When the more frequent stimulus is predicted, the prediction effect should decline slightly, if at all, with increases in PSI; (2) when the less frequent stimulus is predicted, the prediction effect should decrease with increasing PSI; and (3) at the 0-sec PSI, the prediction effect for the less frequent stimulus should equal the prediction effect for the more frequent stimulus (i.e., residual probability effects should be absent at the 0 -sec PSI).

As can be seen in Figure 1, Predictions 1 and 2 were confirmed. Contrary to Prediction 3 , however, residual probability effects were still obtained at a 0-sec PSI.
There are two possible reasons for this finding. First, there are residual probability effects over and above those that are accounted for by the expectancy and the substitution hypotheses. These residual probability effects are perhaps localized in response-selection operations. A second possibility is that a subject's verbal prediction of the less frequent stimulus may not correspond to the subject's preparatory set. Informal questioning of subjects after the session was completed revealed that at times they predicted the less frequent stimulus even though they thought the more frequent stimulus would be presented. At present, however, there is no objective evidence favoring one interpretation over the other.

A competing theoretical interpretation of the results of the present studies is Geller's continuous expectancy hypothesis (Geller \& Pitz, 1970). The main idea behind this hypothesis is that a subject may be partially prepared for a particular stimulus. This notion contrasts with a two-state model (Falmagne, 1965; Lupker \& Theios, 1975, 1977) in which a stimulus is in either a prepared or an unprepared state. Rather than viewing the decline in the magnitude of the prediction effect with increasing PSI as indicative of spontaneous all-ornone changes, a continuous expectancy hypothesis would assume a monotonic decrease in the confidence of a predicted event. There are, however, some problems with the continuous expectancy hypothesis. First, an indicant of continuous expectancy is the subject's confidence rating of his prediction. Confidence ratings have produced mixed effects on RTs to correct and incorrect predictions. Geller and Pitz (1970) reported that confidence ratings had no effect on choice reaction speed. Geller and Whitman (1973), despite considerable procedural differences from the previous study, reported a confidence effect only for correct predictions. These inconsistent results make the continuous expectancy hypothesis, in our opinion, somewhat untenable. Second, Theios' (1973) memory stack version of Falmagne's two-state model has generated useful predictions that are difficult to incorporate into a continuous expectancy interpretation. In a four-stimuli two-response task, Hacker and Hinrichs (1974) required subjects to predict the most likely stimulus as well as the next most likely stimulus. The obtained linear function relating RT and prediction order is consistent with a memory stack interpretation. Acosta (1977), using a two-, four-, and six-stimuli, two-response task, found a linear function relating RT and expected memory stack position.' While these results are consistent with a memory stack interpretation of the verbal prediction effect, they are difficult to incorporate into a continuous expectancy model as delineated by Geller and his associates.

\section{REFERENCES}

Acosta, E., JR. Selective preparation: $A$ comparison of cues and predictions in speeded decision making. Unpublished doctoral dissertation, University of lowa, 1977. 
Bernstein, I. H., \& Reese, C. Behavioral hypothesis and choice reaction time. Psychonomic Science, 1965, 3, 259-260.

FalmaGne, J. C. Stochastic models for choice reaction time with application to experimental results. Journal of Mathematical Psychology, 1965, 12, 77-124.

GeLler, E. S. Preceding prediction outcome and prediction outcome probability: Interacting determinants of choice reaction time. Journal of Experimental Psychology, 1974, 103, 426-430.

Geller, E. S., \& Pitz, G. Effects of prediction, probability, and run length on choice reaction time. Journal of Experimental Psychology, 1970. 84, 361-367.

Geller, E. S., \& Whitman, C. P. Prediction outcome and choice reaction time: A memory dependent relationship. Journal of Experimental Psychology, 1972, 96, 334-337.

Geller, E. S., \& Whitman, C. P. Confidence in stimulus predictions and choice reaction time. Memory \& Cognition, 1973, 1 , 361-368.

Geller, E. S., Whitman, C. P., \& Post, D. S. Stimulus probability and prediction outcome as determinants of choice reaction time: Some procedural considerations. Acta Psychologica, 1973. 37. 1-14.

Hacker, M. J., \& Hinrichs, J. V. Multiple predictions in choice reaction time: A serial memory scanning interpretation. Journal of Experimental Psychology, 1974, 103, 999-1005.

HINRICHS, J. V. Probability and expectancy in two-choice reaction time. Psychonomic Science, 1970, 21, 227-228.

HinRICHS, J. V., \& CRAFT, J. L. Verbal expectancy and probability in two-choice reaction time. Journal of Experimental Psychology. $1971,91,367-371$

Holender. D. \& B Bertelson, P. Selective preparation and time uncertainty. Acta Psychologica, 1975, 39, 193-203.

KEELE. S. W. Repetition effect: A memory dependent process. Journal of Experimental Psychology, 1969, 80, 243-248.
Lupker, S. J., \& Theios, J. Tests of two classes of models for choice reaction times. Journal of Experimental Psychology: Human Perception and Performance, 1975, 1, 137-146.

LUPKER, S. J., \& Theios, J. Further tests of a two-state model for choice reaction times. Journal of Experimental Psychology: Human Perception and Performance, 1977, 3, 496-504.

Posner, M. I., \& Boles, S. J. Components of attention. Psychological Review, 1971, 78, 391-408.

Shiffrin, R. M., \& Schneider, W. An expectancy model for memory search. Memory \& Cognition, 1974, 2, 616-628.

ThEIos, J. Reaction time measurements in the study of memory processes: Theory and data. In G. H. Bower (Ed.), The psychol. ogy of learning and motivation (Vol. 7). New York: Academic Press. 1973.

Whitman, C. P., \& Geller, E. S. Sequential effects of stimulus probability and prediction on choice reaction time. Journal of Experimental Psychology, 1972, 93, 273-278.

Whitman, C. P.. \& Geller, E. S. Prediction outcome probabilities as determinants of choice reaction time. Perception \& Psychophysics, 1973, 13, 105-107.

\section{NOTE}

1. Assuming a serial, self-terminating memory search, correct predictions for all set sizes will be found in the first position of the memory stack. Incorrect predictions for Set Sizes 2, 4, and 6 will be the arithmetic means of the remaining positions, that is, Positions 2, 3, and 4, respectively.

(Revision accepted for publication November 28, 1978.) 